\title{
On the border of medical specialties: ovarian metastasis from colorectal cancer
}

\author{
Katarzyna A. Kowalczyk ${ }^{1,2,30}$, Wojciech M. Wysocki ${ }^{1,2,4}$ \\ ${ }^{1}$ Department of General, Oncological and Vascular Surgery, $5^{\text {th }}$ Military Clinical Hospital in Cracow, Poland \\ ${ }^{2}$ Chair of Surgery, Faculty of Medicine and Health Sciences, Andrzej Frycz Modrzewski Cracow University, Poland \\ ${ }^{3}$ Department of Anatomy, Jagiellonian University Medical College, Cracow, Poland \\ ${ }^{4}$ National Institute of Oncology, Maria Skłodowska-Curie Memorial, Scientific Editorial Office, Poland
}

\begin{abstract}
The colon cancer (CC) is the fourth most common cancer in the world. It is associated with metastatic spread in $50 \%$ of cases in the course of the disease. Common sites for synchronous metastases from colorectal cancer are the lung, liver, peritoneum, bone and brain. The frequency of ovarian metastasis from CC varies widely from 1.6 to $7.4 \%$. This type of metastasis is difficult to distinguish clinically from primary ovarian neoplasms. We present a case of a 49-years old woman admitted to the Department of General Surgery at the $5^{\text {th }}$ Military Clinical Hospital in Cracow for elective surgery for metastatic obstructive sigmoid cancer. Computed tomography (CT) showed a large tumor in the right ovarian field. Brief recommendations regarding that issue based on the available literature has been summarized as well.
\end{abstract}

Key words: colorectal cancer; metastasis; ovarian tumor

Ginekologia Polska 2021; 92

\section{INTRODUCTION}

The colon cancer (CC) is the fourth most common cancer in the world. About 576,000 men and 521,000 women, respectively, are projected to be diagnosed with CC in 2018 . This incidence constitutes a $1.51 \%$ cumulative risk of CC among men aged $0-74$ years, and a $1.12 \%$ risk among women. [1]. CC is associated with metastatic spread in 50\% of cases in the course of disease [2]. Common sites for synchronous metastases from colorectal cancer are the lung, liver, peritoneum, bone and brain [3]. The frequency of ovarian metastasis from CC varies widely from 1.6 to $7.4 \%$ (the rate of ovarian metastases from CC is reported to be up to $30 \%$, but this refers to autopsy patients who died from (C). This type of metastasis is difficult to distinguish clinically from primary ovarian neoplasms - even up to $45 \%$ of CC metastases are clinically mistaken for primary ovarian tumors. The optimal first-line treatment strategy is debatable [3-6].

\section{CASE REPORT}

We present a case of a 49-years old woman admitted to the Department of General Surgery at the $5^{\text {th }}$ Military
Clinical Hospital in Cracow for elective surgery for metastatic obstructive sigmoid cancer.

She was diagnosed with a growing tumor in the mesoand hypogastric area and abdominal pain. Computed tomography $(C T)$ showed a large tumor $(15 \times 11 \times 9 \mathrm{~cm})$ in the right ovarian field (Fig.1). CT also confirmed the presence of the sigmoid wall thickening on the section about $7 \mathrm{~cm}$ long with blurred borders. Furthermore, numerous minor liver lesions were noted, most likely metastatic on imaging.

Colonoscopy revealed in the sigmoid $(18 \mathrm{~cm}$ upwards from the anal sphincter) a circular obstructive infiltrate impervious to the endoscope. Biopsy specimen on histopathology confirmed the diagnosis of adenocarcinoma.

Due to impending bowel obstruction patient was scheduled for primary resection of sigmoid with further systemic therapy afterwards. On admission patient was in good general condition, no vomiting, normosthenic, without signs of cachexia (normal total protein and albumin level); abdominal wall arched at the level of the chest, with tumor palpable in the right iliac fossa, no peritoneal signs and normal peristalsis. Laboratory tests found mild anemia (HGB

\footnotetext{
Corresponding author:

Katarzyna A. Kowalczyk

Department of General, Oncological and Vascular Surgery, $5^{\text {th }}$ Military Clinical Hospital in Cracow, Poland

e-mail: katarzyna.anna.kowalczyk@gmail.com
}

This article is available in open access under Creative Common Attribution-Non-Commercial-No Derivatives 4.0 International (CC BY-NC-ND 4.0) license, allowing to download articles and share them with others as long as they credit the authors and the publisher, but without permission to change them in any way or use them commercially. 




Figure 1. Computed tomography showed a large tumor in the right ovarian field

$10.8 \mathrm{~g} / \mathrm{dL}$ ) and elevated tumor markers (CEA $11 \mathrm{ng} / \mathrm{mL}$, Ca 19-9 $125 \mathrm{IU} / \mathrm{mL}$, Ca 125 362,2 IU/mL).

Intraoperatively, the right and centrally located $25 \times 15 \mathrm{~cm}$ tumor was found, as well as the lower sigmoid tumor of $5-6 \mathrm{~cm}$ in size, significantly narrowing the intestinal lumen. Additionally, multiple neoplastic seeds were noted in the pouch of Douglas and massive metastatic lesions of both liver lobes. Surgery was performed as planned (sigmoid resection with primary anastomosis); additionally, liver metastasis biopsy was done. Remnant of the right ovary and right oviduct extensively infiltrated by the tumor were noted and resected en bloc as well. Clinical diagnosis of metastasis to the right ovary was seen. Postoperative course was uneventful, and the patient was discharged home.

Final histopathological report confirmed the diagnosis of sigmoid cancer (G2 adenocarcinoma pT3 N0 M1a). The infiltration to the right ovary was metastatic, while the sample taken from the liver was non-diagnostic on histopathology.

\section{DISCUSSION}

Apart from primary ovarian malignancies, which warrant appropriate gynecological diagnostic work-up, ovaries are also relatively common site of secondary tumors. According to Yvonne et al., metastasis to the ovary accounted for $15 \%$ of all ovarian malignancies identified and the gastrointestinal tract was the most common primary site (39\%) [11]. During the initial laparotomy, up to $3.4-10.3 \%$ of patients with CC are found to have synchronous metastases to the ovary [6]. An analysis by Segelman et al. [13], (> 3000 patients with (C) reported total incidence rate of synchronous ovarian metastases reaching $1.1 \%$. Main results of this study are shown in Table 1. Metastases are commonly bilateral
Table 1. Incidence of metastases to the ovary in patients with CC according to Segelman et al. [13], 2010

No. of metastases to the ovary in patients with CC (\%)

Synchronous $\quad$ Metachronous

$34 / 3712(1.1 \%) \quad 22 / 1971(1.1 \%)$

CC - colon cancer

on presentation and usually not larger than $10 \mathrm{~cm}$ in its largest dimension $[6,10,11]$ - on the contrary of the case presented here $(15 \mathrm{~cm})$. They are also more commonly seen in premenopausal women [10].

The process by which CC metastasizes more frequently to the ovary versus other intraabdominal organs is still not clearly explained. Some theories suggest hematogenous spread or contact dissemination (migration of malignant cells through peritoneal space) [3].

Hematogenous pathway is convergent with the fact that some authors indicate that a younger premenopausal female which has higher blood flow to the premenopausal ovary present more often with ovarian metastasis $[4,6,8]$.

The optimal treatment for ovarian metastasis from CC depends on the advancement of disease, whether solitary metastasis or multiple foci are present, general status of the patient and numerous other clinical factors. Multidisciplinary approach is mandatory to include at least gynecologists, surgeons and medical oncologists in the decision process. Some recommend surgery for metastatic ovarian lesion if resectable [3]. It is claimed that even if only one ovary is involved, bilateral oophorectomy should be performed, as autopsy series frequently shows bilateral ovarian involvement on the histopathology despite being clinically limited to one ovary only $[6,9]$. The prognosis is generally poor and long-term survival has been reported relatively rare, as ovarian involvement reflects massively advanced disease with microscopic intraperitoneal spread (even if clinically negative on inspection). In CC the detection rate of malignant cells in peritoneal effusion has been reported as $1.4-35.5 \%$. Due to the wide range of cytologic positivity rate for malignant cells in peritoneal effusion, cytologic evaluation of peritoneal fluid is not routinely performed, thus peritoneal fluid has not been regarded as a reliable indicator [12]. The median survival in patients with residual disease after ovariectomy for metastasis is 10 months. $[3,6]$. A better prognosis have females without concomitant peritoneal spread. According to Miller et al., they had median survival time 25.2 months versus 10.8 months $[6,8,10]$. Considering generally poor prognosis some authors suggested palliative surgical management only or emergency surgery, leaving the patient mainly to systemic therapy or best supportive care if not fit enough or with very limited expected survival [6]. 


\section{General remarks on ovarian metastasis from CC}

1. Prophylactic excision and collection of tissue samples of normal-appearing ovaries in patients with CC is neither necessary nor supported by data.

2. Clinical suspicion of ovarian metastasis requires detailed inspection of the entire abdominal cavity in the search of other primary or secondary tumors.

After ruling-out other than ovarian intraabdominal seeds which could not be radically resected/ablated and following radical resection of

3. colon segment with primary focus, unilateral (in premenopausal women, involving the diseased ova-

ry) or bilateral (in postmenopausal women, involving the diseased and the contralateral healthy ovary) ovariectomy is justified.

4. Ovariectomy with metachronic metastases from CC is warranted if primary or secondary excision or ablation of other extragonadal disease lesions is possible.

Different guidelines apply to patients with CC who have hereditary syndromes like HNPCC, Lynch syndrome, Peutz-Jeghers syndrome and

5. BRCA1 and BRCA2 gene mutation-associated syndromes. In this case, the risk of primary ovarian cancer is higher than in the general population, therefore these patients, in addition to treatment of CC, require an appropriate and comprehensive prophylactic plan, including the ovaries.

Based on: Wysocki W. et al. [16], 2013

$\mathrm{CC}$ - colon cancer

Due to impending bowel obstruction, this particular patient was scheduled for primary resection of sigmoid with further systemic therapy afterwards, however in the non-urgent settings an International Ovarian Tumor Analysis protocol is recommended [14].

The concept of prophylactic ovariectomy in patients with CC, described in the past literature, resulted from observed clinical predilection of CC to metastasize to the ovaries. However, there are only a few evidence-based data concerning this issue with conclusions which did not supported the rationale for prophylactic ovariectomy [15]. It seems that prophylactic adnexectomy does not provide any additional benefit $[16,17]$. Brief recommendations regarding that issue based on the available literature has been summarized in the Table 2.

\section{CONCLUSION}

We conclude that a female patient, especially in the premenopausal age with a mass in the ovarian region, should always be also diagnosed for ovarian metastasis deriving from other intraabdominal malignancies. Surgeons and gynecologists who had diagnosed an ovarian metastasis from an unknown origin should keep in mind that the CC is most likely the primary tumor. Multidisciplinary approach is mandatory, as the optimal treatment might encompass gynecologists, surgeons and medical oncologists. Family history taking can be helpful during diagnostic process, with particular focus on hereditary syndromes with increased colon cancer risk - including Hereditary Non Polyposis Colorectal Cancer (HNPCC) and Familial Adenomatous Polyposis (FAP), as well as inflammatory bowel diseases (Crohn's disease, ulcerative colitis) which as well increase the risk of colorectal cancer in younger age women.

\section{Conflict of interest}

None declared.

\section{REFERENCES}

1. Rawla P, Sunkara T, Barsouk A. Epidemiology of colorectal cancer: incidence, mortality, survival, and risk factors. Prz Gastroenterol. 2019; 14(2): 89-103, doi: 10.5114/pg.2018.81072, indexed in Pubmed: 31616522.

2. Jervis MJ, Rodrigues C, Cabral D, et al. Surgical Approach to Colorectal Cancer Pulmonary Metastasis - One-year Experience of a Reference Center. Rev Port Cir Cardiotorac Vasc. 2019; 26(2): 117-119, indexed in Pubmed: 31476811.

3. Shimazaki J, Tabuchi T, Nishida K, et al. Synchronous ovarian metastasis from colorectal cancer: A report of two cases. Oncol Lett. 2016; 12(1): 257-261, doi: 10.3892/ol.2016.4553, indexed in Pubmed: 27347134.

4. Garg S, Dasappa L, Lakshmaiah KC, et al. Colorectal cancer presenting as ovarian metastasis. Clin Cancer Investig J. 2017; 6(1): 92-96, doi: 10.4103/ccij.ccij_14_17.

5. Challa VR, Goud YG, Rangappa P, et al.„,Ovarian Metastases from Colorectal Cancer: Our Experience". Indian J Surg Oncol. 2015; 6(2): 95-98, doi: 10.1007/s13193-014-0369-5, indexed in Pubmed: 26405412.

6. Wright JD, Powell MA, Mutch DG, et al. Synchronous ovarian metastases at the time of laparotomy for colon cancer. Gynecol Oncol. 2004; 92(3): 851-855, doi: 10.1016/j.ygyno.2003.12.017, indexed in Pubmed: 14984952.

7. Mori Y, Nyuya A, Yasui K, et al. Clinical outcomes of women with ovarian metastases of colorectal cancer treated with oophorectomy with respect to their somatic mutation profiles. Oncotarget. 2018; 9(23): 1647716488, doi: 10.18632/oncotarget.24735, indexed in Pubmed: 29662660.

8. Miller BE, Pittman B, Wan JY, et al. Colon cancer with metastasis to the ovary at time of initial diagnosis. Gynecol Oncol. 1997; 66(3): 368-371, doi: 10.1006/gyno.1997.4811, indexed in Pubmed: 9299247.

9. Lee SuJ, Lee J, Lim HoY, et al. Survival benefit from ovarian metastatec tomy in colorectal cancer patients with ovarian metastasis: a retrospective analysis. Cancer Chemother Pharmacol. 2010; 66(2): 229-235, doi: 10.1007/s00280-009-1150-2, indexed in Pubmed: 19820936.

10. Muthukrishnan S, Naganathbabu OL, Murugesan SD, et al. Krukenberg tumours from gastrointestinal cancers-analysis from a tertiary care centre in India. J Gastrointest Oncol. 2018; 9(6): 1164-1167, doi: 10.21037/jgo.2018.07.03, indexed in Pubmed: 30603137.

11. de Waal YRP, Thomas CMG, Oei ALM, et al. Secondary ovarian malignancies: frequency, origin, and characteristics. Int J Gynecol Cancer. 2009; 19(7): 1160-1165, doi: 10.1111/IGC.0b013e3181b33cce, indexed in Pubmed: 19823050

12. Lee InK, Kim DoH, Gorden DL, et al. Prognostic value of CEA and CA 19-9 tumor markers combined with cytology from peritoneal fluid in colorectal cancer. Ann Surg Oncol. 2009; 16(4): 861-870, doi: 10.1245/s10434008-0294-3, indexed in Pubmed: 19189191.

13. Segelman J, Flöter-Rådestad A, Hellborg $H$, et al. Epidemiology and prognosis of ovarian metastases in colorectal cancer. Br J Surg. 2010; 97(11): 1704-1709, doi: 10.1002/bjs.7196, indexed in Pubmed: 20726012.

14. Koneczny J, Czekierdowski A, Florczak M, et al. The use of sonographic subjective tumor assessment, IOTA logistic regression model 1, IOTA Simple Rules and GI-RADS system in the preoperative prediction of 
malignancy in women with adnexal masses. Ginekol Pol. 2017; 88(12): 647-653, doi: 10.5603/GP.a2017.0116, indexed in Pubmed: 29303221.

15. Sielezneff I, Salle E, Antoine K, et al. Simultaneous bilateral oophorectomy does not improve prognosis of postmenopausal women undergoing colorectal resection for cancer. Dis Colon Rectum. 1997; 40(11): 1299-1302, doi: 10.1007/BF02050813, indexed in Pubmed: 9369103.
16. Wysocki W, Kojs Z, Mituś J, et al. Prophylactic ovariectomy in patients with colorectal cancer: is it justified? Current Gynecologic Oncology. 2013; 11(1): 55-61, doi: 10.15557/cgo.2013.0005.

17. Thompson C. Prophylactic oophorectomy during primary colorectal cancer resection: A systematic review and meta-analysis. World Journal of Surgical Procedures. 2015; 5(1): 167, doi: 10.5412/wjsp.v5.i1.167. 